\title{
Efficacy of Parent-Infant-Psychotherapy with mothers with postpartum mental disorder: study protocol of the randomized controlled trial as part of the SKKIPPI project.
}

Janna Mattheß ( $\sigma_{\text {janna.matthess@ipu-berlin.de ) }}$

International Psychoanalytic University Berlin gGmbH https://orcid.org/0000-0001-9111-9530

Melanie Eckert

International Psychoanalytic University Berlin gGmbH

Katharina Richter

Diakonissenkrankenhaus Flensburg

Gabriele Koch

International Psychoanalytic University Berlin gGmbH

Thomas Reinhold

Charite Universitatsmedizin Berlin

Petra Vienhues

Diakonissenkrankenhaus Flensburg

Anne Berghöfer

Charite Universitatsmedizin Berlin

Stephanie Roll

Charite Universitatsmedizin Berlin

Thomas Keil

Charite Universitatsmedizin Berlin

Franziska Schlensog-Schuster

Universitat Leipzig

Kai von Klitzing

Universitat Leipzig

Christiane Ludwig-Körner

International Psychoanalytic University Berlin gGmbH

Lars Kuchinke

International Psychoanalytic University Berlin gGmbH

Study protocol 
Keywords: Randomized controlled trial, parent-infant-psychotherapy, attachment, maternal sensitivity, postpartum mental disorder, inpatient psychiatric department, home visits

Posted Date: January 9th, 2020

DOl: https://doi.org/10.21203/rs.2.20503/v1

License: (c) (1) This work is licensed under a Creative Commons Attribution 4.0 International License. Read Full License

Version of Record: A version of this preprint was published at Trials on June 5th, 2020. See the published version at https://doi.org/10.1186/s13063-020-04443-7. 


\section{Abstract}

Background: After the birth of a child, many mothers and fathers experience postpartum mental disorders like depression, anxiety, obsessive-compulsive disorder, stress or other illnesses. This endangers the establishment of attachment between the children and their primary caregivers. Early problems in parentchild interaction can have adverse long-term effects on the family and the child's well-being. In order to prevent a transgenerational transmission of mental disorders, it is necessary to evaluate psychotherapeutic interventions that target psychologically burdened parents of infants or toddlers. The aim of this trial is to investigate the efficacy of Parent-Infant-Psychotherapy (PIP) for mothers with postpartum mental disorder and their infants (0-12 month).

Methods: In this open randomized controlled intervention trial 180 mother-infant dyads will be included and randomly allocated to 12 sessions of PIP or care as usual. The interventions take place either in inpatient adult psychiatric departments or in outpatient settings with home visits. The primary outcome is the maternal sensitivity assessed by the Sensitivity subscale of the Emotional Availability Scale (EAS) through videotaped dyadic play-interactions after 6 weeks. Secondary outcomes are maternal attachment (AAI) and reflective functioning, psychopathology, maternal stress, infant development and attachment after 6 weeks and 12 months. In addition, resource usage and associated costs will be measured.

Discussion: There is increasing demand for well-controlled studies on psychotherapeutic interventions in the postpartum period that do not only focus on particular risk groups. This RCT represents one of the first studies to investigate the efficacy of PIP in inpatient psychiatric departments and outpatient care centres in Germany. The results will fill knowledge gaps on the factors contributing to symptom reduction in postpartum mental disorders and improvements in mother-child relationships and help developing preventive and therapeutic strategies for the fragmented German health care system.

Trial registration: German Register for Clinical Trials: DRKS00016353.

\section{Background}

Parental mental health problems during infancy are known to impact children's emotional well-being and to modulate future developmental trajectories [1-3]. Postpartum mental health problems show high prevalence rates. In the first year after the birth of a child up to $22 \%$ of all mothers develop a postpartum depression (PPD) [see 4], and nearly $21 \%$ of all women suffer from postpartum anxiety [1] or obsessivecompulsive illnesses [5] with a high comorbidity of these disorders [6]. Parental mental health problems have been identified as major factors for problems in parent-child interaction and as risk factors for the child's well-being [7, 8]. In contrast, a positive mother-child relationship is an essential resilience factor for child development. Maternal sensitivity towards the infant is seen as key variable in mother-child interaction and secure attachment development [9-11]. Particularly in postpartum depressions, a lack of sensitivity and low parental skills are known to affect the interaction with the child which has adverse effects on the child's development [12]. According to Lyons-Ruth [2], these malignant patterns increase 
the risk of disorganized attachment patterns and child psychopathologies [13]. Recent reviews emphasize that this relationship might be more complex and dependent on the severity of the problems and the presence of additional risk factors [14]. Available evidence thus favours interventions that focus on altering maternal or paternal sensitivity which in turn are more effective in enhancing attachment security $[1,4,14]$.

Parent-Infant-Psychotherapy (PIP) has been developed as intervention that aims to improve the attachment relationship between an infant and its parents to pave the way for a healthy child development $[1,15,16]$. PIP aims not only on reducing parental postpartum psychopathological symptoms but also on establishing stable relationship patterns and improving maternal or paternal sensitivity $[15,17,18]$. In comparison to other interventions such as parenting programs, family support and medication [19-21], PIP shows high effect sizes on attachment development [see 15, 22]. Barlow et al. [see 15,22] recently reviewed 8 clinical trials comprising either comparisons of PIP with no-treatment controls or other types of control groups. There was no difference in the efficacy of improving maternal sensitivity between all types of interventions, but PIP was significantly more effective in improving attachment security. While the meta-analysis of Barlow et al. did not reveal differences for measures of parental sensitivity [1], the authors clearly noted that the quality of the included studies was low while heterogeneity of these samples was high. The available evidence seems to indicate that PIP is more effective in subgroups of at-risk populations but more research addressing these limitations is needed [1, $23,24]$.

This seems to be particularly true in case of mothers with mental health problems and young infants. Only four randomized controlled trials (RCTs) examining PIP with infants under 1 year of age have been published [1, 25-27]. These studies clearly differed in the addressed subpopulations (imprisoned mothers [25], depressed mothers [28], social adverse mother [1], diverse maternal concerns and problems [26]) and the investigated settings (prison [25], outpatient setting [26], clinical setting [1], and home visits [28]). Only the RCT of Cooper et al. [28] required that study participants have a diagnosis of PPD as an inclusion criteria, while in the other studies participants had not to fulfil specific diagnoses at the time of recruitment $[25,26]$ or only research-related cut-offs for mental health problems [1]. Only one of these four studies [26] found evidence for an improvement of maternal sensitivity at post-intervention measurements and none revealed effects on infants' attachment. Thus, there is currently neither evidence for the effectiveness of PIP with infants under 1 year of age nor conclusive evidence regarding clinical settings and psychiatric diagnoses. It therefore remains unclear whether this is due to the subpopulations examined, or the diagnostic criteria applied or the setting.

Thus, despite the clear benefits of attachment- and sensitivity-related interventions for the mother-childdyad [19], a corresponding study of PIP with validation in inpatient psychiatric departments is still needed. Moreover, recent developments in child psychiatry provide evidence for the effectiveness of home treatment or home-based interventions [29-31]. Home visits by psychotherapists have the advantage of an intervention in the patient's own environments, which might be particularly helpful for mothers in the post-partum period. It is assumed, that staying in one's own environment and with the familiar daily 
routine, may have a positive influence on the treatment outcome. PIP home-based interventions have demonstrated to effectively improve the children's attachment organization [32], but null effects on attachment measures and maternal sensitivity have been reported as well $[33,34]$. Again, there are large methodological differences between these studies.

In summary, previous randomized trials investigated the positive effect of PIP on the attachment quality of infants (and long-term effects up to preschool age [35]). In contrast to the present study, most of these studies have focused exclusively on specific risk groups or have taken place in outpatient settings and home visits but with relatively low diagnostic cut-offs $[1,25,26,28,34]$. The efficacy of PIP in inpatient departments and home-based interventions for mothers with diagnosed postpartum mental disorder will be investigated in this randomized controlled efficacy study.

\section{Methods And Design}

\section{Overview}

The SKKIPPI research project (www.skkippi.de) is a multicentre research project in Germany located in Berlin, Flensburg, Hamburg and Leipzig with the aim to examine epidemiology and treatment of psychosocial and mental disorders in the postpartum period. SKKIPPI is divided into an epidemiological cohort study that evaluates the prevalence of psychosocial and mental disorders in the postpartum period, the utilisation of health and social care services, and their economic burden, and two randomized controlled intervention studies to evaluate the efficacy of PIP.

\section{Aims and hypotheses}

The present RCT is designed to evaluate the efficacy of focused PIP interventions in inpatient adult psychiatric departments with Mother and Baby Units (MBU) or in outpatient psychiatric departments or via home visits. The decision about setting (inpatient or outpatient) depends on the severity of the symptoms and/or maternal consent. It is hypothesized that in comparison to routine therapy (care as usual, $\mathrm{CAU}$ ) mothers with mental disorders and their infants under 1 year of age will benefit from an increase in maternal sensitivity after 6 weeks of intervention. It is expected that the routine therapy also reduces the psychopathological symptoms, but nevertheless has little or no effect on maternal sensitivity.

Secondary hypotheses

1. a) In comparison to routine therapy, PIP is effective in reducing maternal psychopathological symptoms and psychological distress for mothers with mental disorders in the post-partum period.

2. b) In comparison to routine therapy, PIP enhances reflective functioning in mothers with mental disorders in the post-partum period.

3. c) In comparison to routine therapy, PIP enhances the rate of securely attached mother-child-dyads at 12 months follow-up. 
Subgroup analyses are made between PIP and CAU depending on the setting (inpatient compared to outpatient/ home visits). In addition, health economic analyses will examine the use of health and care services in Germany by mothers with mental disorders in the postpartum period.

\section{Trial design}

The study is designed as a two-arm, open, randomized, controlled, multicentre trial with parallel groups and blinded endpoint assessment (PROBE design; prospective randomized open blinded endpoint) with 6 weeks of intervention.

Depending on the severity of the psychopathological symptoms and in agreement with the participants' preferences, the decision for a particular treatment setting (inpatient or outpatient) will be made before randomization. After assignment to treatment setting, participants will be randomly allocated to either the PIP intervention group or CAU control group. Randomization will be carried out independently by a biometrician of the as a 1:1 block randomization with variable block length and stratified by setting (inpatient or outpatient) and study centre. Treatment allocation is masked to the assigning personnel. Data of mothers and children will be assessed at three measurement points (see Fig. 1): at baseline after randomization and immediately before the intervention (T0), after the intervention at 6 weeks (T1), and at 12-month follow-ups (T2). Study centres are located in the German cities Berlin and Flensburg. At the time of writing additional effort is made for a study centre in Hamburg.

\section{Participants}

A sample of 180 mother-child-dyads is planned to participate in the study. After screening for eligibility, written informed consent and the decision about treatment setting, the participants will be randomly allocated to either the PIP intervention arm (planned $n=90$ ) or the CAU control arm (planned $n=90$; see Fig. 2). Mother-child-dyads will receive monetary compensation of $€ 100$ to take part in the study until the 12month follow-up.

Inclusion criteria are that the participants will be German-speaking mothers with an ICD-10 diagnosis of a mental disorder in the postpartum period and their infants under 1 year of age. Diagnoses will be made by certified therapists at the clinical study centres.

Exclusion criteria are a maternal ICD-10 diagnosis of schizophrenia, substance abuse or recent suicidal ideation. Infants will be excluded when they show symptoms of alcohol embryopathy or severe chronic organic disease. Mothers and infants will be excluded when they are participants in further clinical trials or undergo another psychotherapy.

Participants are recruited predominantly via health visiting services and mental health services, through links with community services, and advertisements in midwife practices and paediatricians. 


\section{Intervention}

Focused Parent-Infant-Psychotherapy is a psychotherapeutic intervention in which mother-child-dyads are treated together. Individual sessions could also be held together with the father. PIP aims at fostering the parent-infant-relationship by supporting the parents' ability to understand and mentalize the child's affective states. It aims at promoting parental self-reflection and sensitivity to support the building and maintaining of parent-infant-relationships. PIP interventions are exclusively delivered by trained and supervised psychotherapists who are additionally certified in the PIP method. The manualized PIP [36] is a focused psychodynamic intervention with 12 succeeding sessions of approx. 50 minutes in 6 weeks (2 sessions per week). Aims of PIP are clarification of problems, affect regulation, self-reflection, a change of perspective and the activation of the participants' interpersonal resources. The intervention includes a mentalization-based approach, and works with reframing, an appropriate confrontation within a supportive framework, psychoeducation, the development of action strategies and video-feedback. To enhance manual adherence, all study therapists are additionally trained in the manual before the start of the treatment.

The inpatient treatment is characterized by interventions in adult psychiatric departments with MBUs or day hospitals. In inpatient settings, PIP will be provided in addition to standard therapeutic care. In outpatient settings PIP is provided in the patients' home environment or in psychiatric outpatient departments.

\section{Control}

Participants in the CAU group benefit from the standard (social) pedagogical and therapeutic care provided by the German health system over the intervention period of 6 weeks. Due to ethical considerations, no 'waiting list' group was implemented. CAU therefore resamples a heterogeneous class of standard interventions. As a result, more intense and therapeutic care might be provided for motherchild-dyads in the inpatient settings compared to standard care in the outpatient setting. CAU in the inpatient setting could include counselling, body or occupational therapy and other psychiatrictherapeutic interventions for the participant. The frequency of these interventions is generally lower as PIP treatment. CAU in outpatient setting could be other therapeutic interventions or (social) pedagogical care such as developmental psychological counselling or physiotherapy. Because this might result in heterogeneities in the number and intensity of CAU, all individual interventions will be documented.

\section{Assessment and outcome measures}

\section{Primary endpoint}

Maternal Sensitivity. Primary outcome of the current study is maternal sensitivity at the end of 6-week intervention (T1) measured by the Emotional Availability Scale (EAS). EAS [37] is a widely used 
assessment for the quality of mother-child interaction with excellent psychometric properties. Short videotaped dyadic play interactions are assessed and coded by independent and reliable coders blind to treatment allocation. EAS is assessed at all 3 measurement time-points and coded using a Likert Scale (1 to 7) on maternal sensitivity as well as three further maternal dimensions (structuring, non-intrusiveness and non-hostility) and two child dimensions (responsiveness to parent and involvement with parent).

\section{Secondary endpoints}

Child attachment. The most important secondary outcome is child attachment measured by the SSP [38]. SSP is a standardized procedure of eight episodes to record the quality of child's attachment. The aim of SSP is to activate the child's attachment system. The test is recorded on video and will be coded by reliable and independent coders blind to treatment allocation. This results in the classification of the quality of infants' attachment behaviour as being secure (B), avoidant (A), or ambivalent (C). In addition, coders are also trained to classify disorganized (D) attachment qualities. Because SSP can only be validly assessed starting at an age of 11 months, it will only be measured at the 12-month follow-up timepoint. Group differences in child attachment will be examined using binarized SSP classification (secure/ insecure).

Because no cut-off values are available, dichotomous response variables for the EAS sensitivity scale at $\mathrm{T} 1$ and $\mathrm{T} 2$ will be computed using the Reliable Change Index (RCl) as additional secondary endpoints $(\mathrm{RCl}>1.96$, see [39]).

Further secondary outcomes for the maternal psychopathological symptoms:

M.I.N.I. - The Mini International Neuropsychiatric Interview (M.I.N.I.) [40] is a structured diagnostic interview which assesses 20 psychiatric disorders by DSM IV and ICD-10 diagnosis. The M.I.N.I. is used to verify the clinical diagnoses and will be administered by trained interviewers at T0 and T2.

BSCL - The Brief-Symptom-Checklist (BSCL) [41] is a validated questionnaire for assessing maternal psychopathological symptoms. The BSCL will be measured at T0 and T1.

EPDS - The Edinburgh Postnatal Depression Scale (EPDS) [42] is a standard screening instrument to measure maternal symptoms of postnatal depression. The EPDS will be assessed at all 3 measurement time-points. Dichotomous response variables will be computed according to the cut-offs reported in [42] $($ EPDS < 12).

ASQ - Maternal symptoms of anxiety disorder will be measured by the Anxiety Screening Questionnaire (ASQ) [43]. The ASQ is a questionnaire which assesses all anxiety disorders diagnosed by DSM IV and ICD-10. It will be measured at T0 and T1.

IES-27 - For assessing Borderline Personality Disorder, the Scale for impulsive behaviour and emotional dysregulation of Borderline Personality Disorder (IES-27) [44] is used. The IES is a screening instrument 
which measures typical behavioural and perceptual patterns of this disorder. The questionnaire will be assessed at $\mathrm{T} 0$ and $\mathrm{T} 1$.

PRFQ-1 - The Parental Reflective Functioning Questionnaire (PRFQ) [45] is a validated questionnaire which measures the mother's ability to mentalize in context of the relationship to her child. The questionnaire will be assessed at all 3 measure times. Because no cut-off values are available, dichotomous response variables for the PRFQ will be computed based on the $\mathrm{RCI}>1.96$ criterion [39].

PSI - The German version of the Parenting Stress Index (PSI) $[46,47]$ is a questionnaire which measures the maternal stress at T0, T1 and T2. Because no cut-off values are available, dichotomous response variables for the PSI will be computed based on the $\mathrm{RCI}>1.96$ criterion [39].

AAI - Maternal attachment style and reflective functioning, i.e. the capacity to mentalize, are considered as important moderators in the present design and are assessed using the Adult Attachment Interview (AAI) [38]. AAl is a semi-structured interview with excellent psychometric properties to evaluate the maternal 'state of mind' with respect to attachment [38]. The AAI will be conducted by trained interviewers at baseline (TO), transcribed, and subsequently scored by reliable and independent coders blind to treatment allocation.

Secondary outcome measures for the child are:

SFS - The infant's symptoms are measured by using the German questionnaire Crying, Feeding, Sleeping (SFS) [48]. The SFS assesses problems in the child's behavioural regulation and dysfunctional communication patterns, and stress symptoms of the primary caregivers. The questionnaire will be assessed at $\mathrm{T} 0$ and $\mathrm{T} 1$.

CBCL - To detect the child's emotional and behavioural problems, the Child-Behavior-Checklist (CBCL) [49] is used. The CBCL is validated at a child's age of 18 months and will be therefore assessed at 12-month follow-up only.

ET-6-6-R - The level of social-emotional, cognitive, motor and language development of the child is assessed by the German development test ET6-6-R [50]. The ET-6-6-R is a standardized test for children aged from six months to six years that measures the developmental status of the child. The test will be measured at $\mathrm{T} 1$ and $\mathrm{T} 2$.

Further secondary outcomes

Treatment Adherence is measured using an in-house developed short 30 item Treatment Adherence Questionnaire. PIP therapists are asked to evaluate after each session the methods and interventions applied.

Health Care and Social Services Utilisation and Costs. To estimate economic consequences on the health care service usage in Germany, a cost analysis will be carried out by using a self-developed questionnaire. 
Questions about the utilisation of health and social care and demand on early intervention services are assessed. The questionnaire will be applied at T0 and T2.

\section{Samples size calculations and statistical analyses}

A power analysis [G*Power] based on a reported moderate to large effect size of Cohen's $d=0.64$ for a difference in maternal sensitivity after 6 weeks of intervention between intervention group and CAU group [19] and two-tailed testing with a significance level of $a=5 \%$ using an ANCOVA (with maternal sensitivity at T1 as the dependent variable and maternal sensitivity at T0 as a covariate) resulted in a needed sample size of $n=70$ (per group) to detect an effect with a power of $95 \%$. With an assumed drop-out rate of about $20 \%$ [1] the sample size calculates to $n=90$ per group (total sample size $n=180$ mother-childdyads).

The statistical analysis is carried out at the end of the last follow up assessment and all analyses will be conducted in accordance with intention-to-treat (ITT). principles and will be including all randomized

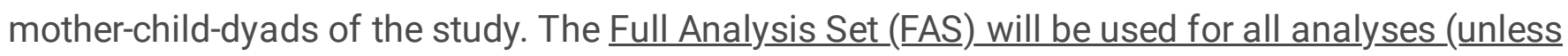
otherwise stated), it includes all randomized mother-child-dyads. Data will be analysed according to randomization irrespectively of the treatment received. Data from mother-child-dyads who have withdrawn from the study will be included in the FAS up to the date of their termination. The Per Protocol (PP)_population comprises a subset of the FAS. It consists of mother-child-dyads who received treatment according to the randomization, have adequate therapy adherence, and no major protocol violations. Major protocol violations are considered as not meeting all inclusion criteria, a change of treatment group or as PIP intervention is not carried out properly according to the treatment manual. The safety. population includes mother-child-dyads who have participated in at least one intervention session. Participants in the safety population will be analysed according to the treatment actually received.

Primary analysis of the primary outcome. The evaluation of the primary outcome (EAS, maternal sensitivity at T1) is performed by a covariance analysis (ANCOVA) with the independent variable treatment (PIP vs. CAU), adjusted for maternal sensitivity at baseline T0 and treatment setting as stratification variable based on the FAS population. Adjusted mean values of treatment groups with $95 \%$ confidence intervals and p-value (two-sided) for group comparisons are presented.

Secondary analyses of the primary outcome. The primary analysis will be repeated as described with the PP population. In addition, the effect of treatment setting on the efficacy of PIP will be investigated by means of a moderator analysis. Moderators show their effect in the interaction with treatment group. Thus, the model will include all variables as described for the primary analysis with the addition of the treatment*setting interaction. Additional moderator analyses regarding the effectiveness of PIP including maternal attachment style and reflective functioning (AAI) as moderators will be calculated. A sensitivity analysis of the primary outcome will be computed using multiple imputation methods and the PP population adjusted for maternal sensitivity at baseline and treatment setting as stratification variable. In a further sensitivity analysis of the primary outcome, a hierarchical regression model will be calculated 
which, in addition to the adjustment for the baseline values and the treatment setting, includes the therapists who performed the intervention in the model. Both sensitivity analyses will be repeated using also study centre as stratification variable.

Linear Mixed Models for longitudinal data will be used to examine the change of the primary outcome across the measurement time points for the treatment groups. The model term will include predictors for treatment group and time as fixed effects as well as the treatment group*time interaction, baseline maternal sensitivity as covariate, treatment setting as stratification variable, and subjects as random effects. The therapists and further predictors will be added to the model when indicated by goodness-offit model comparisons (based on AIC values). All secondary analyses of the primary and secondary outcomes are considered exploratory.

Analyses of the secondary outcomes. Secondary outcomes will be computed according to the analysis strategy of the primary outcome using ANCOVA or logistic regression (depending on the scale of the respective outcome, continuous or dichotomous). Likely moderators will be added to the models including setting, and maternal attachment style and reflective functioning. In order to investigate the longitudinal course until the 12-month follow-up, linear mixed models will also be calculated for the change of secondary outcomes (e.g. EPDS, PSI and PRFQ). In addition, sensitivity analyses will be performed for the health economic parameters.

Missing data. Analyses of primary and secondary endpoints will be conducted based on the FAS population without imputation of missing data. A sensitivity analysis is computed by imputing missing data of the primary outcome with multiple imputation methods for linear regression using MCMC simulations. The selection of imputation variables will be decided blinded to treatment allocation and at least 5000 iteration and 100 imputations will be used.

\section{Methods to minimize bias}

Data quality is continuously monitored, and the study supervised by the Institute for Social Medicine, Epidemiology and Health Economics of the Charité - Universitätsmedizin Berlin (SEGC) to keep the researchers and outcome raters blinded for treatment group allocation. Data on the intervention will be unblinded after the last participant finished the study. Only pseudonymous data sets will be analyzed. All participants are randomly allocated to treatment based on a computer generated randomization list by an independent biometrician of the SEGC.

The study sets a high standard in PIP treatment and minimizes therapeutic bias insofar that all study therapists are trained in the manual before the beginning of treatment and are under supervision by experienced PIP therapists. For monitoring the therapeutic process, all therapists will complete the treatment adherence questionnaire after each treatment session. To minimize bias for the assessments, most of the data will be assessed electronically and stored in a secured database supervised by the SEGC. In addition, the study has a strict separation of research assessments and therapeutic intervention. 
Staff members responsible for data collection are blind for randomization and treatment group allocation and research assistants are well trained in assessment methods.

\section{Ethical considerations and limitations}

Data will be assessed, processed, and stored according to the data protection laws and the local data protection protocol. The research project will be carried out in compliance with the study protocol and the Declaration of Helsinki (1996 version, Somerset West). All researchers and participants in the study are obliged to follow the rules of Good Clinical Practice (ICH-GCP). Participants will only be enrolled in the study after given inform consent. The study is designed as an open, randomized controlled trial with blinded endpoint assessment and blinded analysis. Still, participants are aware of their treatment group allocation.

This trial compares a psychotherapeutic intervention with available care as usual interventions. CAU interventions will be heterogeneous and administered under very diverse conditions. Therefore, it will be difficult to document which component of CAU is most effective. Moreover, there will be differences in CAU depending on the setting (inpatient vs. outpatient). Due to ethical concerns participants will not be randomly allocated to a setting. Thus, more severe cases will be evident in the inpatient setting lowering the representativeness of the results. At the same time CAU routine intervention will be more intense in inpatient departments, i.e. CAU in inpatient settings will likely include other (psycho-)therapeutic interventions. Furthermore, ethical consideration precluded the inclusion of a waiting list group. This very same concerns will also lead to the fact that all mother-child-dyads allocated to CAU will be offered an intervention or care. Thus, the standard in the CAU will likely be higher than in the overall population in Germany which will bias generalizability of the results. In order to minimize these effects and to incorporate these limitations in the analyses, all interventions in all conditions will be documented.

There is a lack of good quality trials for the proof of efficacy of PIP in treatment of mothers [15] but more specifically of fathers [24]. This present study is designed for mothers with postpartum mental disorders. The importance of the fathers' involvement for treatment outcomes, for the family system and the impact on child's development is not assessed, neither will father-infant-dyads be included in the study. Following the study by Letourneau $[51,52]$ mothers with PPD report that fathers are a key resource for support. Up till now, little is known about fathers' effect on the system, and with the present design no conclusions regarding fathers can be drawn.

A further limitation of this study is the eligibility of a diverse range of maternal postpartum diagnoses which will likely lower the power to detect effects. On the other hand, this design was chosen because of the higher generalizability when not restricting inclusion criteria to single diagnoses. If sufficient data are available, exploratory analyses of subsamples with the same diagnoses are intended. The PIP intervention in the present study has been designed as a short-term treatment of 12 sessions for parentinfant-dyads. Short sensitivity-based interventions have been shown to have superior efficacy compared 
with longer ones $[15,19]$. Still, in case of highly traumatized mother it might be less effective. Of note is also that study therapists and participants will not be blind to treatment allocation.

Some studies [23,53-55] point out, that there is a link between the engagement of participant, withdrawal and therapeutic alliance. In this trial, therapeutic alliance and patients' motivation will not be assessed. But all withdraws and severe events will be documented and contributed to the statistical analyses.

\section{Discussion}

Interventions with a focus on attachment and maternal sensitivity and in particular parent-infant psychotherapeutic interventions have good evidence base for the treatment of mental health problems in the postpartum period $[15,19]$ and the foundation of a healthy child development. Given the high prevalence of postpartum mental disorders for mothers and the adverse impact on the child, a highquality study focusing on the efficacy of PIP in Germany is needed. PIP in inpatient psychiatric departments has not been in the focus of controlled trials so far, and the present study differs to previous ones in that no particular risk samples are examined (like social adverse samples [1] or imprisoned mothers [25]) but diagnosed mothers with mental disorders in the postpartum period. There is a strong focus on prevention studies in early childhood in the German health care services. For the particular case of mental disorders, the trial is expected to reveal the efficacy of PIP in comparison with CAU in inpatient psychiatric settings and also in outpatient home visits to contribute to the ongoing discussion in the German health care system. As a further result, it will be expected to provide information for practitioners, and mothers and fathers on factors contributing to the efficacy of PIP interventions and to improve future care situation for patients and their families in Germany.

\section{Trial status}

The recruitment for the trial started in January 2019 and is ongoing (as of November 2019). It is expected to be completed in September 2021. This protocol is version 1.0, dated 13 September 2018.

\section{Declarations}

\section{Ethics approval and consent to participate}

Central ethical approval has been confirmed from the ethics committee of the International Psychoanalytic University Berlin (reference number: 2018-16) covering local ethics approval for the centres in Berlin and Flensburg and the study will not begin recruiting at other centres in the trial until local ethical approval has been obtained. Written informed consent will be obtained from all participants and their legal guardians to participate in the study. At any time, participants may withdraw their consent for any reason without any negative consequences regarding their treatment.

\section{Consent for publication}


Not applicable.

\section{Availability of data and materials}

Not applicable.

\section{Competing interests}

The authors declare that they have no competing interests.

\section{Funding}

This study as part of SKKIPPI project is supported by a grant from the Innovationsfonds/GBA Germany (No 01 VSF17027). The sponsor had no role in the design of the study and will not have any role during its execution, analyses, interpretation of the data, or decision to publish results.

\section{Acknowledgements}

Not applicable

\section{Authors' contributions}

LK, CLK, PV, KvK and TK wrote the grant application and drafted the manuscript together with JM, ME, KR, GK, AB, SR, TR and FSS. JM, LK, CLK and SR designed the trial with the help of FSS, KvK, GK, AB and ME. CLK, GK, LK and PV supervised the implementation of the study. SR and LK calculated the sample size and planned the statistical analyses. JM, ME, KR and GK acquire the data. JM, ME and GK provide the training. All authors read and approved the final manuscript.

\section{Abbreviations}

SKKIPPI - [acronym] Parent-Infant-Psychotherapy in cohort- and intervention studies [Eltern-S̈äugling-

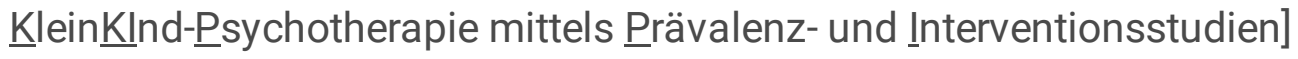

PIP - Parent-Infant-Psychotherapy

RCT - randomized controlled intervention trial

CAU - care as usual

PPD - postpartum depression 
MBU - Mother and Baby Units

AAI - Adult Attachment Interview

PRFQ - Parental Reflective Functioning Questionnaire

SSP - Strange Situation Procedure

PROBE design - Prospective randomized open blinded endpoint

EAS - Emotional Availability Scale

M.I.N.I. - Mini International Neuropsychiatric Interview

BSCL - Brief-Symptom-Checklist

EPDS - Edinburgh Postnatal Depression Scale

ASQ - Anxiety Screening Questionnaire

IES-27 - Scale for impulsive behaviour and emotional dysregulation of Borderline Personality

PSI - Parenting Stress Index

SFS - German questionnaire Crying, Feeding, Sleeping [Schreien, Füttern, Schlafen]

CBCL - Child-Behavior-Checklist

ET6-6-R - German development test

ANCOVA - Analysis of covariances

ITT - Intention-to-treat

FAS - Full Analysis Set

PP - Per Protocol

$\mathrm{RCl}$ - Reliable Change Index

AIC - Akaike Information Criterion

MCMC - Markov chain Monte Carlo simulations

SEGC - Institute for Social Medicine, Epidemiology and Health Economics of the Charité

ICH-GCP - Rules of Good Clinical Practice 
DRKS - German Register for Clinical Trials [Deutsches Register Klinischer Studien]

\section{References}

1. Fonagy P, Sleed M, Baradon T. Randomized Controlled Trial of Parent-Infant-Psychotherapy for Parents with Mental Health Problems and Young Infants. Infant Mental Health Journal. 2016;37(2):97-114.

2. Lyons-Ruth K. Contributions of the mother-infant relationship to dissociative, borderline, and conduct symptoms in young adulthood. Infant Mental Health Journal. 2008;29(3):203-18.

3. Oates M. Perinatal psychiatric disorders: a leading cause of maternal morbidity and mortality. British Medical Bulletin. 2003;67:219-29.

4. Cox JL, Murray D, Chapman G. A Controlled Study of the Onset, Duration and Prevalence of Postnatal Depression. British Journal of Psychiatry. 1993;163:27-31.

5. Fairbrother $\mathrm{N}$, Young AH, Janssen $\mathrm{P}$, Antony MM, Tucker E. Depression and anxiety during the perinatal period. BMC Psychiatry. 2015;15(1):206.

6. Reck C, Struben K, Backenstrass M, Stefenelli U, Reinig K, Fuchs T, et al. Prevalence, onset and comorbidity of postpartum anxiety and depressive disorders. Acta Psychiatrica Scandinavica. 2008;118(6):459-68.

7. Kindler H. [How could a risk inventory for early intervention look like?] Wie könnte ein Risikoinventar für Frühe Hilfen aussehen? In: Meysen T, Schönecker L, Kindler H, editors. Frühe Hilfen im Kinderschutz. Weinheim, München: Juventa; 2009. p. 170-261.

8. Tronick E, Reck C. Infants of depressed mothers. Harv Rev Psychiatry. 2009;17(2):147-56.

9. Ainsworth MDS, Bell SM, Stayton DJ. Infant-mother attachment and social development: Socialisation as a product of reciprocal responsiveness to signals. In: Richards MPM, editor. The integration of a child into a social world. Cambridge: Cambridge University Press; 1974. p. 99-135.

10. Bowlby J. Attachment and Loss Vol. 1. 2 ed. New York: Basic Books; 1982.

11. Grossmann KE, Grossmann K, editors. [Attachment and human development. John Bowbly, Mary Ainsworth and the foundation of attachment theory] Bindung und menschliche Entwicklung. John Bowbly, Mary Ainsworth und die Grundlagen der Bindungstheorie. Stuttgart: Klett-Cotta; 2003.

12. Zwönitzer A, Ziegenhain U, Bovenschen I, Pillhofer M, Spangler G, Gerlach G, et al. [Early support and children's cognitive development: A longitudinal pilot study of psychosocial burdened mother-child dyads in early childhood] Frühe Hilfen und kindliche kognitive Entwicklung: Eine längsschnittliche Pilotuntersuchung psychosozial belasteter Mutter-Kind-Paare in der frühen Kindheit. Prax Kinderpsychol Kinderpsychiat. 2016;65(5):340-53.

13. Stein A, Pearson RM, Goodman SH, Rapa E, Rahman A, McCallum M, et al. Effects of perinatal mental disorders on the fetus and child. The Lancet. 2014;384(9956):1800-19.

14. Wan MW, Green J. The impact of maternal psychopathology on child-mother attachment. Arch Womens Ment Health. 2009;12(3):123-34. 
15. Barlow J, Bennett C, Midgley N, Larkin SK, Wei Y. Parent-infant psychotherapy: a systematic review of the evidence for improving parental and infant mental health. Journal of Reproductive and Infant Psychology. 2016;34(5):464-82.

16. Winberg Salomonsson M. Long-term effects of mother-infant psychoanalysis. Stockholm: Karolinska Instituet; 2017.

17. Ludwig-Körner C. [Parent-Infant-Toddler-Psychotherapy] Eltern-Säuglings-Kleinkind-Psychotherapie. Göttingen: Vandenhoeck \& Ruprecht; 2016.

18. Ludwig-Körner C. [Parent-Infant-Toddler-Psychotherapy] Eltern-Säuglings-Kleinkind-Psychotherapie. In: von Sydow K, Borst U, editors. Systemische Therapie in der Praxis. Weinheim: Beltz Verlag; 2018.

19. Bakermans-Kranenburg MJ, Van IJzendoorn MH, Juffer F. Less is more: Meta-analyses of sensitivity and attachment interventions in early childhood. . Psychological Bulletin. 2003;129(2):195-215.

20. Euser S, Alink LRA, Stoltenborgh M, Bakermans-Kranenburg MJ, van ljzendoorn MH. A gloomy picture: a meta-analysis of randomized controlled trials reveals disappointing effectiveness of programs aiming at preventing child maltreatment. BMC Public Health. 2015;15:1068.

21. Taubner S, Wolter S, Rabung S. Effectiveness of early-intervention programs in German-speaking countries - a meta-analysis. Mental Health \& Prevention. 2015;3(3):69-78.

22. Letourneau N, Tryphonopoulos P, Giesbrecht G, Dennis C-L, Bhogal S, Watson B. Narrative and metaanalytic review of intervention aiminig to improve maternal-child attachment security. Infant Mental Health Journal. 2015;36(4):366-87.

23. Ransley R, Sleed M, Baradon T, Fonagy P. “What support would you find helpful?" The relationship between treatment expectations, therapeutic engagement, and clinical outcomes in parent-infant psychotherapy. Infant Mental Health Journal. 2019;40(4):557-72.

24. Lumsden V. Does the evidence support parent-infant psychotherapy?: Commentary on... Cochrane Corner. BJPsych Advances. 2017;23(4):217-21.

25. Sleed M, Baradon T, Fonagy P. New Beginnings for mothers and babies in prison: A cluster randomized controlled trial. Attachment \& Human Development. 2013;15(4):349-67.

26. Salomonsson B, Sandell R. A randomized controlled trial of mother-infant psychoanalytic treatment: II. Predictive and moderating influences of qualitative patient factors. Infant Ment Health J. 2011;32(3):377-404.

27. Murray L, Cooper PJ, Wilson A, Romaniuk H. Controlled trial of the short-and long-term effect of psychological treatment of post-partum depression: 2. Impact on the mother-child relationship and child outcome. The British Journal of Psychiatry. 2003;182(5):420-7.

28. Cooper PJ, Murray L, Wilson A, Romaniuk H. Controlled trial of the short-and long-term effect of psychological treatment of post-partum depression: I. Impact on maternal mood. The British Journal of Psychiatry. 2003;182(5):412-9.

29. Mattejat F, Hirt B, Wilken J, Schmidt M, Remschmidt H. Efficacy of inpatient and home treatment in psychiatrically disturbed children and adolescents. European child \& adolescent psychiatry. 2001;10(1):S71. 
30. Boege I, Corpus N, Schepker R, Kilian R, Fegert JM. Cost-effectiveness of intensive home treatment enhanced by inpatient treatment elements in child and adolescent psychiatry in Germany: A randomised trial. European Psychiatry. 2015;30(5):583-9.

31. Böge I, Schepker R, Fegert JM. [Visiting forms of treatment for mentally ill children and adolescents] Aufsuchende Behandlungsformen für psychisch kranke Kinder und Jugendliche. Bundesgesundheitsblatt-Gesundheitsforschung-Gesundheitsschutz. 2019;62(2):195-204.

32. Cicchetti D, Rogosch FA, Toth SL. Fostering secure attachment in infants in maltreating families through preventive interventions. Dev Psychopathol. 2006;18(3):623-49.

33. Lieberman AF, Weston DR, Pawl JH. Preventive Intervention and Outcome with Anxiously Attached Dyads. Child Development. 1991;62(1):199-209.

34. Murray L, Cooper PJ, Wilson A, Romaniuk H. Controlled trial of the short- and long-term effect of psychological treatment of post-partum depression. 2 Impact on the mother-child relationship and child outcome. 2003;182(5):420-7.

35. Winberg Salomonsson M, Sorjonen K, Salomonsson B. A Long-Term Follow-Up of a Randomized Controlled Trial of Mother-Infant Psychoanalytic Treatment: Outcomes on the Children Infant Mental Health Journal. 2015;36(1):12-29.

36. Schlensog-Schuster F, Koch G, Ludwig-Körner C. [SKKIPPI-Studymanual for Parent-Infant-ToddlerPsychotherapy (PIP)] SKKIPPI-Studienmanual für Eltern-Säugling-Kleinkind-Psychotherapie (ESKP). [Unpublished Manual]. In press.

37. Biringen Z, Derscheid D, Vliegen N, Closson L, Easterbrooks MA. Emotional availability: Theoretical Background, empirical research using the EA Scales, and clin-ical applications. Developmental Review. 2014;34:114-67.

38. Ainsworth MDS, Blehar MC, Waters E, Wall S. Patterns of attachment: A psychological study of the strange situation. New York: Earlbaum; 1978.

39. Jacobson NS, Truax P. Clinical significance: a statistical approach to defining meaningful change in psychotherapy research. Journal of consulting and clinical psychology. 1991;59(1):12-9.

40. Sheehan DV, Lecrubier Y, Harnett-Sheehan K, Janavs J, Weiller E, Bonara LI, et al. Reliability and Validity of the M.I.N.I. International Neuropsychiatric Interview (M.I.N.I.): According to the SCID-P. Eur Psychiatry. 1997;12:232-41.

41. Franke GH. Brief-Symptom-Checklist (BSCL). Manual. Göttingen: Hogrefe-Verlag; 2017.

42. Cox L, Holden MH, Sagovsky R. Detection of postnatal depression. Development of the 10-item Edinburgh Postnatal Depression Scale. The British Journal of Psychiatry. 1987;150(6):782-6.

43. Wittchen HU, Boyer P. Screening for anxiety disorders: sensitivity and specificity of the anxiety screening questionnaire (ASQ-15). Br J Psychiatry Suppl. 1998;173:10-7.

44. Kröger C, Kosfelder J. [Scale for impulsive behaviour and emotional dysregulation of Borderline Personality Disorder] Skala zur Erfassung der Impulsivität und emotionalen Dysregulation der Borderline-Persönlichkeitsstörung (IES-27). Manual. . Göttingen: Hogrefe-Verlag; 2011. 
45. Luyten P, Mayesc LC, Nijssens L, Fonagy P. The Parental reflective functioning questionnaire: Development and preliminary validation. PLOSE ONE. 2017;12(5).

46. Abidin RR. Parenting stress index (4th ed.). Lutz, FL: Psychological Assessment Resources; 2012.

47. Tröster H. [Parenting Stress Index (PSI)] Eltern-Belastungs-Inventar (EBI). Deutsche Version des Parenting Stress Index (PSI) von R.R. Abidin. Manual. Göttingen: Hogrefe; 2011.

48. Groß S, Reck C, Thiel-Bonney C, Cierpka M. [Questionnaire Crying, Feeding and Sleeping] Fragebogen zum Schreien, Füttern und Schlafen. 2007.

49. Aschenbach TM, Rescorla LA. Manual for the ASEBA Preschool Forms \& Profiles. In: University of Vermont RCfC, Youth, \& Families, editor. Burlington, VT2000.

50. Petermann F, Macha T. [Development test for children at the age of 6 month to 6 years] Entwicklungstest für Kinder im Alter von 6 Monaten bis 6 Jahren - Revision (ET 6-6-R). Frankfurt a. M.: Pearson Assessment; 2013.

51. Letourneau N, Duffett-Leger L, Stewart M, Hegadoren K, Dennis CL, Rinaldi CM, et al. Canadian mothers' perceived support needs during postpartum depression. J Obstet Gynecol Neonatal Nurs. 2007;36(5):441-9.

52. Letourneau NL, Dennis CL, Benzies K, Duffett-Leger L, Stewart M, Tryphonopoulos PD, et al. Postpartum depression is a family affair: addressing the impact on mothers, fathers, and children. Issues Ment Health Nurs. 2012;33(7):445-57.

53. Fluckiger $\mathrm{C}$, Del Re AC, Wampold BE, Horvath AO. The alliance in adult psychotherapy: A metaanalytic synthesis. Psychotherapy (Chic). 2018;55(4):316-40.

54. Uckelstam C-J, Holmqvist R, Philips B, Falkenström F. A relational perspective on the association between working alliance and treatment outcome. Psychotherapy Research. 2018:1-10.

55. Martin DJ, Garske JP, Davis MK. Relation of the therapeutic alliance with outcome and other variables: a meta-analytic review. Journal of consulting and clinical psychology. 2000;68(3):438-50.

\section{Figures}




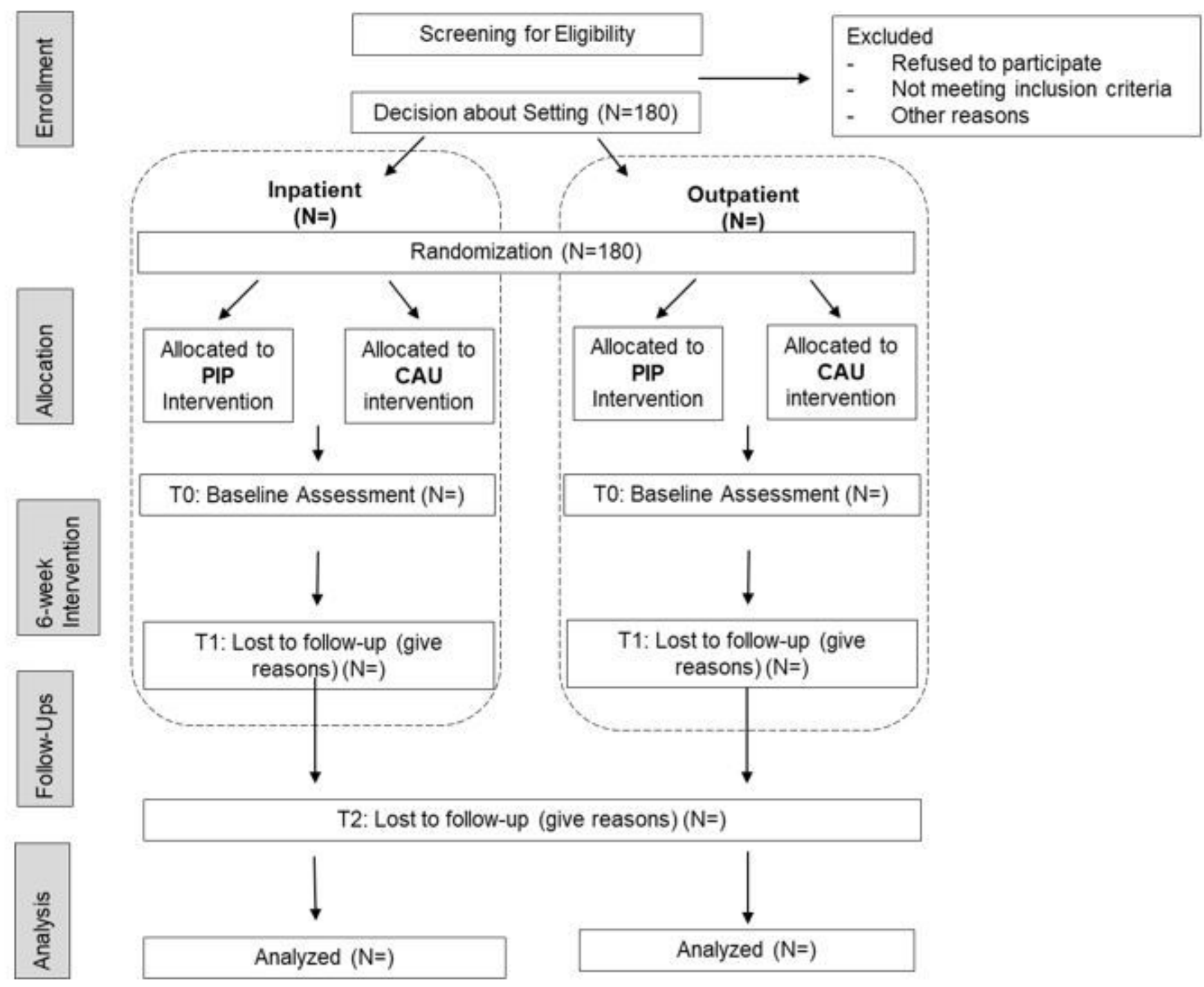

\section{Figure 1}

CONSORT diagram describing flow of mother-child-dyads through the study. PIP Parent-Infant Psychotherapy, CAU Care-as-usual. 


\begin{tabular}{|c|c|c|c|c|c|}
\hline \multirow[b]{3}{*}{ TIMEPOINT } & \multicolumn{5}{|c|}{ STUDY PERIOD } \\
\hline & \multirow{2}{*}{$\begin{array}{c}\begin{array}{c}\text { Enrol- } \\
\text { ment }\end{array} \\
-t_{1}\end{array}$} & \multirow{2}{*}{$\begin{array}{c}\text { Allocation } \\
0\end{array}$} & \multicolumn{3}{|c|}{ Post-allocation } \\
\hline & & & $\begin{array}{c}t_{0} \\
\text { a-weeks }\end{array}$ & $\begin{array}{c}t_{1} \\
\text { e- weoks }\end{array}$ & $\begin{array}{c}t_{2} \\
\text { 12-mann }\end{array}$ \\
\hline \multicolumn{6}{|l|}{ ENROLMENT: } \\
\hline \multirow{3}{*}{$\begin{array}{r}\text { Eligibility screen } \\
\text { Informed consent } \\
\text { Assignment to } \\
\text { treatment setting } \\
\text { (inpatient/ outpatient) }\end{array}$} & $\mathrm{X}$ & & & & \\
\hline & $\mathrm{X}$ & & & & \\
\hline & $\mathrm{X}$ & & & & \\
\hline Allocation & & $\mathrm{X}$ & & & \\
\hline \multicolumn{6}{|l|}{ INTERVENTIONS: } \\
\hline \multirow{2}{*}{$\begin{array}{l}\text { [PIP Intervention] } \\
\text { [CAU Intervention] }\end{array}$} & & & $\leftarrow$ & $\rightarrow$ & \\
\hline & & & $\leftarrow$ & $\rightarrow$ & \\
\hline \multirow{2}{*}{$\begin{array}{l}\text { ASSESSMENTS: } \\
\text { Mother-Child- } \\
\text { Interaction (EAS) }\end{array}$} & & & & & \\
\hline & & & $\mathrm{x}$ & $\mathrm{x}$ & $\mathrm{x}$ \\
\hline M.I.N.I. & & & $\mathrm{X}$ & & $\mathrm{X}$ \\
\hline$B S C L$ & & & $\mathrm{X}$ & $\mathrm{X}$ & \\
\hline EPDS & & & $\mathrm{X}$ & $\mathrm{X}$ & $\mathrm{X}$ \\
\hline$A S Q$ & & & $\mathrm{X}$ & $\mathrm{X}$ & \\
\hline IES-27 & & & $\mathrm{X}$ & $\mathrm{X}$ & \\
\hline$P R F Q-1$ & & & $\mathrm{X}$ & $\mathrm{X}$ & $\mathrm{X}$ \\
\hline PSI & & & $\mathrm{X}$ & $\mathrm{X}$ & $\mathrm{X}$ \\
\hline$A A I$ & & & $\mathrm{X}$ & & \\
\hline SFS & & & $\mathrm{X}$ & $\mathrm{X}$ & \\
\hline$C B C L$ & & & & & $\mathrm{X}$ \\
\hline \multirow{2}{*}{$\begin{array}{c}E T-6-6-R \\
\quad S S P\end{array}$} & & & & $\mathrm{X}$ & $\mathrm{X}$ \\
\hline & & & & & $\mathrm{X}$ \\
\hline $\begin{array}{l}\text { Health Care and So- } \\
\text { cial Services Utilisa- } \\
\text { tion and Costs }\end{array}$ & & & $\mathrm{x}$ & & $\mathrm{x}$ \\
\hline
\end{tabular}

\section{Figure 2}

Full Standard Protocol Items: Recommendations for Interventional Trials (SPIRIT) figure. EAS Emotional Availability Scale, M.I.N.I. - Mini International Neuropsychiatric Interview, BSCL - BriefSymptom-Checklist, EPDS - Edinburgh Postnatal Depression Scale, ASQ - Anxiety Screening Questionnaire, IES-27 - Scale for impulsive behaviour and emotional dysregulation of Borderline Personality, PRFQ-1 - Parental Reflective Functioning Question-naire, PSI - Parenting Stress Index, AAI - 
Adult Attachment Interview, SFS - German ques-tionnaire Crying, Feeding, Sleeping, CBCL - Child-BehaviorChecklist, ET6-6-R - German development test, SSP - Strange Situation Procedure.

\section{Supplementary Files}

This is a list of supplementary files associated with this preprint. Click to download.

- SPIRITFillablechecklistSKKIPPI.doc 